\title{
Fecal microbiota transplantation research output from 2004 to 2017: a bibliometric analysis
}

\author{
Yan Li ${ }^{1,2}$, Ziyuan Zou ${ }^{3}$, Xiaohui Bian ${ }^{2}$, Yushan Huang ${ }^{2}$, Yanru Wang ${ }^{4}$, Chen Yang ${ }^{3}$, Jian Zhao ${ }^{2}$, Lang \\ Xie Corresp. $1^{1}$ \\ ${ }^{1}$ Department of General Surgery, Zhujiang Hospital of Southern Medical University, Guangzhou, Guangdong, China \\ 2 The Second School of Clinical Medicine, Southern Medical University, Guangzhou, Guangdong, China \\ 3 The First School of Clinical Medicine, Southern Medical University, Guangzhou, Guangdong, China \\ 4 Department of Radiation Oncology, Zhujiang Hospital, Southern Medical University, Guangzhou, Guangzhou, China \\ Corresponding Author: Lang Xie \\ Email address: langxiezj@hotmail.com
}

Background: Fecal microbiota transplantation (FMT) is an emerging therapy against Clostridium difficile infection (CDI) and inflammatory bowel disease (IBD). Although the therapy has gained prominence, there has been no bibliometric analysis of FMT. Methods: Studies published from 2004 to 2017 were extracted from the Science Citation Index Expanded. Bibliometric analysis was used to evaluate the number or cooperation network of publications, countries, citations, references, journals, authors, institutions and keywords. Results: A total of 796 items were included, showing an increasing trend annually. Publications mainly came from 10 countries, led by the US $(n=363)$. In the top 100 articles ranked by the number of citations (range 47-1158), American Journal of Gastroenterology (2017 IF $=10.231$ ) took the top spot. The co-citation network had 7 cocitation clusters headed by 'recurrent Clostridium difficile infection'. The top 7 keywords with the strongest citation bursts had three parts, 'microbiota', ' diarrhea ', and 'case series'. All keywords were divided into four domains, 'disease', 'nosogenesis', 'trial', and 'therapy'. Conclusions: This study shows the research performance of FMT from 2004 to 2017 and helps investigators master the trend of FMT, which is also an ongoing hotspot of research. 
1 Fecal microbiota transplantation research output from 2004 to 2017: a

\section{2 bibliometric analysis}

3

4 Yan $\mathrm{Li}^{1,2 *}$, Ziyuan Zou ${ }^{3 *}$, Xiaohui Bian², Yushan Huang ${ }^{2}$, Yanru Wang ${ }^{4}$, Chen Yang ${ }^{3}$, Jian Zhao ${ }^{2}$,

$5 \quad$ Lang Xie ${ }^{1}$

$6 \quad *$ These authors contributed equally to this work.

7

$8{ }^{1}$ Department of General Surgery, Zhujiang Hospital, Southern Medical University, Guangzhou

9 510280, Guangdong, China.

102 The Second School of Clinical Medicine, Southern Medical University, Guangzhou 510515,

11 Guangdong, China.

123 The First School of Clinical Medicine, Southern Medical University, Guangzhou 510515,

13 Guangdong, China.

$14{ }^{4}$ Department of Radiation Oncology, Zhujiang Hospital of Southern Medical University, 15 Guangzhou 510280, Guangdong, China.

17 Correspondence Author

Lang Xie, langxiezj@hotmail.com 
ABSTRACT

24

Background: Fecal microbiota transplantation (FMT) is an emerging therapy against Clostridium difficile infection (CDI) and inflammatory bowel disease (IBD). Although the therapy has gained prominence, there has been no bibliometric analysis of FMT.

Methods: Studies published from 2004 to 2017 were extracted from the Science Citation Index Expanded. Bibliometric analysis was used to evaluate the number or cooperation network of publications, countries, citations, references, journals, authors, institutions and keywords.

Results: A total of 796 items were included, showing an increasing trend annually. Publications mainly came from 10 countries, led by the US $(n=363)$. In the top 100 articles ranked by the number of citations (range 47-1158), American Journal of Gastroenterology (2017 IF = 10.231) took the top spot. The co-citation network had 7 co-citation clusters headed by 'recurrent Clostridium difficile infection'. The top 7 keywords with the strongest citation bursts had three parts, 'microbiota', 'diarrhea', and 'case series'. All keywords were divided into four domains, 'disease', 'nosogenesis', 'trial', and 'therapy'.

Conclusions: This study shows the research performance of FMT from 2004 to 2017 and helps investigators master the trend of FMT, which is also an ongoing hotspot of research.

\section{INTRODUCTION}

41 Fecal microbiota transplantation (FMT) is a therapeutic method by infusing fecal suspensions from 42 a healthy individual into the gastrointestinal tract(Kelly et al., 2016). Zhang et al. noted that GE 
43 Hong pioneered the use of feces to treat human diseases in the Eastern Jin Dynasty (300 400

44 AD) (Zhang et al., 2012). FMT has received public attention over the past decade because of its highly effective treatment of Clostridium difficile infection (CDI) and inflammatory bowel disease (IBD) (Khoruts et al., 2016; Khoruts et al., 2015). In 2013, FMT was included in the CDI treatment guidelines which clearly stipulated that FMT should be considered for patients with a third recurrence of CDI (Surawicz et al., 2013). It was pointed out that recurrent CDI was difficult to treat, and the failure rate of antibiotic therapy was relatively high. Moreover, it has been reported that more than 300 cases of recurrent CDI were effectively treated using FMT (van Nood E et al., 2013). FMT not only has a remarkable cure rate, but is also a safe and acceptable treatment option (Brandt et al., 2012a). However, little systematic analysis of FMT has been performed (Hourigan et al., 2015).

Systematic reviews and meta-analyses are being increasingly used to summarize medical literature and identify areas in which research is needed (Crowther et al., 2010). Unfortunately, they fail to include all the relevant research, meanwhile, the definition of clinical endpoint for the use in combined statistics is unclear and they have publication bias. Quantitative studies of the literature have been performed for nearly one hundred years, during which bibliometric methods have been developed and matured (Rousseau, 2014). Traditional bibliometric methods had been used to evaluate the variations of particular areas by assessing the productivity of countries, institutions, authors and journals (Keathley-Herring et al., 2016). Nowadays, bibliometric analysis provides a statistical and quantitative analysis of publications and offer a convenient way to visibly measure researchers' efforts in the investigation of a specific field (Ashok et al., 2016; Yao et al., 
64 2018).

65 In the current study, we analyzed those were quoted above and provided additional analysis 66 including keywords of those studies and the clusters of citations(Choi \& Kim, 2018; Miao et al.,

67 2017a; Suk et al., 2011). Multiple analytical tools were used to map the trends of FMT research

from 2004 to 2017. This analysis will assist researches in understanding the literature regarding

FMT, and determining the future directions for future study of FMT.

\section{MATERIALS \& METHODS}

\section{Search strategy}

Data were acquired from the Science Citation Index Expanded (SCI-E) of the Web of Science Core Collection (WoSCC) of Clarivate Analytics (https://clarivate.com/) on April 22, 2018. The data were downloaded from the WoSCC so that there were no ethical issues. The searching included literature published from 2004 to 2017, and used the following keywords and terms: 'Fecal bacteria transplantation\$' or 'Intestinal Microbiota Transfer\$' or 'Fecal Transplantation\$' or 'Fecal Transplant\$' or 'Donor Feces Infusion\$'. All electronic searches were performed on the same day, April 22, 2018, to avoid changes in citation rates. The year 2018 was excluded because database entries for the year would not be complete at the time of the search. When all data were collected, the results were arranged according to the 'Times cited'.

\section{Study selection}

83 Two independent reviewers (Y. Li and Y.S. Huang) collected all the data by reading the titles and abstracts acquired from SCI-E of the WoSCC database. When necessary, the full text was 
downloaded from PubMed or other databases. Articles were included only if the main topic was FMT, and the language was English. Exclusion criteria were: (1) the main topic of the article was not about FMT; (2) the abstract of the article couldn't be acquired from WoSCC; (3) the article was a duplicate. Any differences between the two reviewers were settled through discussion with a third reviewer.

\section{Assessment of the articles and journals}

Two researchers (Y.S. Huang and J. Zhao) reviewed the selected articles, and the following data were identified and recorded for analysis: (1) titles, (2) authors, (3) citation number, (4) keywords, (5) publication year, (6) topics (7) funding and (8) countries of origin. Furthermore, the journal names and impact factors (IFs) were also recorded using the 2017 edition of the Journal Citation Reports (JCR).

\section{Statistical analysis}

Data were converted to txt format and imported into CiteSpaceV, GraphPad Prism 5 , the Online Analysis platform of Literature Metrology (http://bibliometric.com/) and VOSviewer. Data were then analyzed cooperation network of keywords, institutions, cited reference, and authorship quantitatively and qualitatively by CiteSpaceV. GraphPad Prism version 5.0 was used to evaluate the strength and direction of the linear relations between the number of citations in the top 100 (T100) cited articles and the number of years since publication, the number of authors, the number of institutions, and the number of countries. It was also used to analyze the correlation of article 
106 citations between different databases (WoSCC and Scopus). All probability values were two-

107 tailed, and the threshold for significance was set at $P<0.05$. Using the Online Analysis platform

108 of Literature Metrology, the number of included articles and the number of articles according to

109 country, published each year were reported.. The analysis also showed the number of the top 17

110 keywords in each year. Exhibiting the relative positions and density of nodes in a network, two

111 dimensional knowledge maps can be produced by VOSviewer which is a computer program

112 primarily intended to be used for mapping, analyzing and exploring different types of networks

113 (Su \& Lee, 2010).

\section{RESULTS}

Total numbers of published items

117 It was absolutely necessary to consider the number of published items on FMT as an index of research productivity. From 2004 to 2017, a total of 2,062 publications on FMT were identified through our search strategy in WoSCC, and 824 papers were screened out according to their titles and abstracts, 1,238 publications were excluded because they did not meet our exclusion criteria ( titles uncorrelated to FMT in 809 papers, the main contents of 125 articles were irrelevant to FMT, the abstracts were inaccessible for 302 papers, and 2 papers duplicated with other articles). Moreover, 28 articles were excluded because they are non-English language. Thus, 796 articles on FMT from 2004 to 2017 were included in the analysis (Figure 1). One article was published in $2004(n=1)$, and it was in $2011(n=15)$ and $2012(n=31)$ when the numbers of published articles each year began to increase dramatically. The yearly number of published articles reached a peak 
127 in $2017(\mathrm{n}=248)$ (Figure 2(A)).

128

129 Distribution by countries

130 The 796 publications on FMT were primarily published by ten countries/regions $(\mathrm{n}=722,90.70 \%)$

131 (Figure 2(B)). The greatest number of publications came from the United States $(\mathrm{n}=363,50.28 \%)$,

132 followed by China (72, 9.97\%), Canada (69, 9.56\%), and Germany (41, 5.68\%). The United States

133 was the only country with a dynamic growth in the number of published articles over last five

134 years. An analysis of international cooperation was shown in Figure 2(C); the most frequent collaboration was between the United States and Canada, followed by the US and UK.

Distribution by citations

Of the 796 selected articles, the top 100 articles ranked by the number of citations (Table 1,

Supplementary Table S1). The median number of citations was 95 (range 47-1158), and three

papers were cited over 500 times. The citation index (median 21.17, range 7.18-231.60) was

correlated with the number of citations $\left(\mathrm{r}^{2}=0.83, P<0.01\right)$ per article in the Web of Science

database. In addition, the number of citations and citations index per article was strongly correlated

in the Scopus database (Figure 3: $\mathrm{r}^{2}=0.84, P<0.01$ ) (Supplementary Figure S1). The T100 articles

were published from 2007 to 2016, with the most articles published in $2015(\mathrm{n}=22)$, followed by

$2013(n=20$; Figure 3). Interestingly, the number of citations in $2013(n=4,136)$ was the highest,

followed by $2015(n=2774)$.

To identify factors that might influence the number of citations of the T100 articles, we 
148 analyzed possible correlations between the number of citations and the number of countries,

149 funding, years since publication, and authors using GraphPad Prism 5 (Figure 3). There was a

150 strong correlation between the number of citations and the number of authors $\left(\mathrm{r}^{2}=0.05, P<0.05\right)$.

151 However, no significant correlations were present between the number of citations and the number

152 of countries $\left(\mathrm{r}^{2}=0.01, P=0.46\right)$, institutions $\left(\mathrm{r}^{2}=0.01, P=0.27\right)$, and years since publication $\left(\mathrm{r}^{2}\right.$ $153=0.01, P=0.27)$.

\section{Analysis of references}

156

157

158

159

160

161

162

163

164

165

166

167

168

Analysis of cited references is crucial to bibliometric analysis, as the scientific relevance of publications can be evaluated by the map of co-cited references. Using CiteSpaceV, the co-citation network was divided into 7 co-citation clusters (Figure 4(A)). The modularity Q score was 0.54 , and the silhouette score was 0.32 . These clusters were labeled by index terms from their own citers. The largest cluster was cluster \#0 labeled as 'recurrent Clostridium difficile infection,' followed by cluster \#1, labeled as 'Clostridium difficile infection.' (Supplementary Table S2)

\section{Distribution by journals}

The 796 articles were published by 294 journals. The top 100 were from 52 journals (Table 2, Supplemental Table 3). According to the Journal Citation Reports (JCR) 2017 standards, the American Journal of Gastroenterology (2017 IF = 10.231) made contributions to the largest number of articles on FMT (10 articles, 10.00\%), followed by Gastroenterology $(2017$ IF = 20.773; 8 articles, 8.00\%), Journal of Clinical Gastroenterology (2017 IF = 2.968; 8 articles, 8.00\%), and 
169 Clinical Infectious Diseases (2017 IF $=9.117 ; 4$ articles, 4.00\%).

170

171 Distribution by authors

172 There were more than 3,000 authors who contributed to the publications on FMT. The network 173 maps of the authors and co-cited authors produced by CitespaceV are shown in Figure 4(B)\& (C).

174 Kassam $Z$ had the greatest number of articles $(n=33)$, followed by Khoruts A $(n=32)$ and Kelly 175 CR $(n=21)$. The top five cited authors were Khoruts A $(n=755)$, by Brandt LJ $(n=720)$, de Vos

WM $(n=557)$, and Nieuwdorp M ( $\mathrm{n}=509)$ (Table 3\&4).

\section{Distribution by institutions}

The publications on FMT were from 933 institutions, and extensive cooperation network analysis

was carried out between institutions (Figure 4(D)). The top five institutions ranked by the number

of articles published 253 articles, about $31.78 \%$ of the total. The University of Texas MD

Anderson Cancer Center $(\mathrm{n}=9,12.44 \%)$ published the greatest number, followed by the

University of Washington $(\mathrm{n}=58,7.23 \%)$, McMaster University $(\mathrm{n}=34,4.27 \%)$, Emory

University $(n=32,4.02 \%)$, and the University of Alabama at Birmingham $(n=30,3.77 \%)$ (Table

$5)$.

Knowledge map of FMT research

Figure 5 illustrates a two-dimensional knowledge map with major keywords as nodes from 2004

to 2017. It shows the frequency of keywords and their relative co-occurrence with colors of 
190

191

192

193

194

195

196

197

198

199

200

201

202

203

204

205

206

207

208

209

210

different intensity to represent it as a heat map. Intense (blue) color indicates the frequency of

keywords. A keyword labelled in larger font size turns up in the center of each intense color, which

indicates that these keywords appeared more frequently and co-occurred with a higher number of

other keywords in the literature. In addition, network visualization of keywords was shown in

Figure S2, the distance between two keywords in the visualization approximately indicates the relatedness of the keywords in terms of co-citation links. In general, the closer two keywords are

located to each other, the stronger their relatedness. The strongest co-citation links between keywords are also represented by lines.

\section{Analysis of kewords}

An approximation of research trends was found by analyzing the top 17 keywords (Figure 2(D)).

Almost all the keywords appeared rising and falling fluctuations but an ascendant trend. The

keywords of 'fecal microbiota transplantation' and 'Clostridium difficile' were the first and second

most frequent in the last five years of the study period. Except for searching keywords which were

'fecal microbiota transplantation, 'fecal transplantation', and 'facal transplant', the three most

frequent keywords were 'Clostridium difficile', 'microbiome', and 'inflammatory bowel disease'.

These three words are also the main therapeutic directions of all FMT researches in the world.

The top 7 keywords with the strongest citation bursts were extracted by CiteSpaceV (Table

6). The blue line represents the time interval and the red line represents the duration of a burst keyword, suggesting the beginning and the end of the time interval of each burst. The top keyword was 'flora' (10.56, 2011-2014), followed by 'bacteriotherapy' $(7.69,2009-2013)$ and 'diarrhea' 
$211(7.02,2011-2013)$.

212 There were 722 different keywords in the 796 publications. All keywords with the same 213 meaning were merged into one keyword. After data standardization, 481 keywords were selected 214 as core keywords. Among them, 59 keywords appeared more than three times whose frequency of 215 occurrence were $70.24 \%$ so that they were selected as core keywords. The classification results in 216 Table 7 are shown in four domains: disease, nosogenesis, trial, and therapy. Among the 59 core 217 keywords, the highest percentage was in the disease domain $(40.49 \%)$, followed by the therapy 218 domain $(41.44 \%)$, the nosogenesis domain $(14.63 \%)$ and the trial domain $(3.77 \%)$. The 219 transplantation topic in the therapy domain contained the highest percentage $(33.30 \%)$ of core

220 221 222 223 224 225 226 227 228 229 230

keywords, which were 'fecal microbiota transplantation' 'microbiome' 'stool transplantation' 'bacterial consortium transplantation' 'bacteriotherapy' 'gut microbiome transplantation' and 'transplantation'. In the disease domain, infection topics $(16.79 \%)$ occurred most frequently.

Microbiota topics (11.13\%) occurred most frequently in the nosogenesis domain. The subject topic in the trial domain accounted for $2.42 \%$.

DISCUSSION

\section{Total number of published items}

28 This was the first bibliometric analysis of FMT. The yearly number of publications rapidly increased from one article in 2004 to 248 articles in 2017, with an average annual increase of $17.35 \%$ over the last 5 years of the study period. Compared with the total scientific output in the WoSCC database, its average annual growth rate was 3.84\% between 2004 and 2017. The 
232

233

234

235

236

237

238

239

240

241

242

243

244

245

246

247

248

249

250

251

252

increasing incidences of CDI and IBD might explain the increase in the numbers of publication from 2004 to 2017 (Rohlke \& Stollman, 2012; Kelly et al., 2012). In addition, the increasing number of output index in the WoSCC database might have also made contribution to the increase in the number of publications. A conclusion can be drawn from the rapid development of FMT, whose research was only in its infancy but promising and potential.

\section{Country of origin and institutions}

The top 10 countries/regions in which FMT studies were performed accounted for for $90.70 \%$ of the total number of publications. China was the only developing country among these 10 countries/regions, indicating that China has made great progress in the FMT research recently. It is not surprising that developed countries produced the greatest numbers of publications (Van Rossum et al., 2010; Halpenny et al., 2010). The United States, which produced 363 publications (45.60\%), demonstrated a leading role in FMT research. This might be related to the high incidence of CDI and IBD in the United States (Orenstein et al., 2013). It might also be related to the financial resources devoted to scientific study in the United States (Zhao et al., 2016). In addition, the United States played a key role in promoting international cooperation, with the strongest international cooperation between the United States and Canada, followed by between the United States and the United Kingdom (Figure 2C). As regard to the cooperation of institution, the top five institutions were ranked by the number of articles, among which 4 institutions subordinated to the United States. This conclusion was consistent with the dominance of the United States. A conclusion could be drawn that the increasing incidence of CDI and IBD has promoted research of FMT in 
253

254

255

256

257

258

259

260

261

262

263

264

265

266

267

268

269

270

271

272

273

this field.

\section{Citation count and possible factors influencing citations}

Based on the clarity of network structure and clustering, two indexes were proposed by CitespaceV: the modularity Q score and the mean silhouette score. Modularity and silhouette metrics provided useful quality indicators of clustering and network decomposition. A low modularity means that a network can't be reduced to a cluster with clear boundaries, while a high modularity suggests a well-structured network. The silhouette metric is helpful for estimate the uncertainty involved in identifying the nature of a cluster (Chen et al., 2010). The co-citation network was divided into 7 co-citation clusters with a modularity of 0.54 and a silhouette of 0.32 . This result suggested, the inter-cluster connections are considerable, but not overwhelming and are diverse and heterogeneous.

Citation counting, a proxy measure of research quality, can help authors understand the characteristics inherent in highly cited studies and provide a new perspective on specific areas (Yan et al., 2011; Fu \& Aliferis, 2008). However, there has been no citation analysis of FMT until now. Of 796 selected articles, we focus on the top 100 articles arranged by the number of citations.

Interestingly, there was no output in 2017. It's likely that 'older' articles have a longer citable period and attained more citations, and as such accumulative citation frequency will be higher (Liu et al., 2016). To further examine this, we assessed the correlation between years since publication and citation count. However, no obvious correlation was found. In addition, we replaced citation count with citation index to decrease the effect of publication time. Citation counts are quantitative, 
274 they can be added, subtracted, normalized, and ploted, satisfactorily, citation index based on these 275 counts abound, the ubiquitous Hirsch or h-index being the most prominent (Will, 2014), and the

276 h-index is an author-level metric that attempts to measure both the productivity and citation impact

277 of the publications of a scientist or scholar (Prathap, 2012). The results indicated that citation count

278 and citation index exhibited a substantial correlation, indicating that publication time has very little

279 influence on citations. In addition, we analyzed the citations of the top 100 publications in Scopus.

280 A strong relationship was found between citation index in WoSCC and citation index in Scopus,

281 so does the relationship between citations and citation index in Scopus, which ruled out the impact 282 of the database on citations.

\section{Journals}

The IFs of journals were one of the strongest indicators for citations in some way owing to the attraction of high IF journals to the scientific community (Garfield, 2003). The top-cited articles are usually published in high IF journals. The top 100 articles ranked by the number of citation were published in 52 journals, with the exception of one, because its IF was not found in the JCR. The median IF of these publications was 6.96, and the Ifs of 18 (35.29\%) publications were greater than 10.00. These results suggested that it was challenging to publish articles on FMT in high IF journals. Meanwhile, it implied the quality of output in this subject area.

\section{Authorship}


294 Ranked by the number of articles they owned, the top five authors identified in this analysis 295 published at least 22 articles. They were consequently regarded as 'prolific authors'. The top five 296 authors contributed to at least 472 citations. Surprisingly, there was one author in both analyses 297 where this author not only did well in the number of publications but also the quality of

298

299

300

301

302

303

304

305

306

307

308

309

310

311

312

313

314

publications. The co-cited authors who had at least 200 co-citation counts, included Van Nood E and Borody TJ. Although neither of them belonged to the category of prolific authors, they played pivotal roles in FMT research; particularly, Van Nood E, who was also the first author of the article with the highest number of citations.

\section{Knowledge map of FMT research}

A total of 243 items were divided into four clusters, cluster 1, 2, 3, and 4 comprised of 122, 144, 9, and 1 items, respectively (Figure $5 \&$ S2). In the item density visualization, each point has a color that indicates the density of items at that point. Colors in the map range from blue to yellow, the larger the number of items (i.e., clostridium difficile infection) in the neighborhood of a point and the higher the weights of the neighboring items, the closer the color of the point is to blue. In the network visualization, items are represented by their label and by default also by a circle. The size of the label and the circle of an item is determined by the weight of the item. The higher the weight of an item, the larger the label and the circle of the item. The color of an item is determined by the cluster to which the item belongs, for example, microbiota in green belong to cluster 2 . Lines between items represent links, meanwhile, the distance between two keywords in the visualization approximately indicates the relatedness of the keywords in terms of co-citation links. 
315 In general, the closer two keywords are located to each other, the stronger their relatedness. The

316 strongest co-citation links between journals are also represented by lines.

\section{Keywords and research fields}

In recent years, there has been great interest in the use of FMT for therapy of gastrointestinal and non-gastrointestinal diseases, mainly for CDI and IBD. In most countries/regions, the incidence of

IBD is increasing or at a relatively high stable level. Furthermore, IBD patients have a nearly threefold higher risk for CDI compared with the general population (Rodemann et al., 2007).

Nevertheless, some patients became refractory to standard therapy and suffered from a poor quality refractory, or severe CDI and IBD, at least when standard treatments had failed (Anderson et al., 2012; Kelly et al., 2014a). The goal of this bibliometric analysis was to provide an overview of FMT's research, and guide future studies.

According to the 59 core keywords identified, 4 domains were classified. The disease domain

had the highest percentage and the trial domain the lowest. Within the disease domain, the topics

of infection and inflammatory have the highest ratio. In this regard, it might relate to the therapy

principle of FMT. Evidence has suggested that there is a reduced diversity of luminal microbiota and increased mucosal adherent bacteria in IBD and CDI(Nagalingam \& Lynch, 2012). Changes in gut microbiota led to a serious imbalance of host physiology and immune homeostasis, which ultimately results in infection and inflammation. Brandt et al (Brandt et al., 2012b) noted that FMT 
gastrointestinal microbiota's role in driving disorders. Interestingly, the topic of emotion taken up

337 a certain proportion, which was an indication that FMT could be used to treat mental illness.

In the therapy domain, the topic of transplantation occupied the largest portion and other

major treatments of IBD and CDI were also listed. In general, the standard therapy for CDI and

IBD included steroids, amino-salicylates, immunosuppressants, and various biological therapies,

most of which are not effective and place a heavy economic burden on patients (Talley et al., 2011;

Kappelman et al., 2008). Some patients become refractory to standard management and suffer significant adverse side effects with a poor quality of life (Mcfarland, 2005). Konijeti et al

(Konijeti et al., 2014) indicated that FMT was the most cost-effective initial strategy for

management of recurrent CDI. FMT can thus reduce the financial burden of patients, and result in

a substantial increase in quality of life (Merlo et al., 2016).

The domains mentioned above clarified research hotspots clear, and burst keywords can be

considered indicators of research frontiers over time (Yin et al., 2016). The blue line represents

the time interval and the red line represents the duration of a burst keyword. There were 7 keywords

with the strongest citation bursts. After taking similar burst keywords into consideration, they were

divided into three categories: (i) Microbiota: The presence of normal, healthy, intestinal microbiota

was now considered to offer protection against CDI and IBD. However, the repeated use of

immunosuppressants and other therapy with drugs severely disrupted the normal gut microbiota,

which always led to the recurrence of CDI and IBD. Instead, FMT allowed the rapid reconstitution

of a normal composition of microbial communities. The wonderful effect and desperate need in 
357 (ii) Diarrhea: Antibiotic-associated colitis caused by Clostridium difficile was the most common

358

359

360

361

362

363

364

365

366

367

368

369

370

371

372

373

374

375

376

377

cause of hospitalization for diarrhea (Cohen et al., 2015). It was caused by disrupting the normal gut flora and led to dysbiosis that enables Clostridium difficile colonization of the patients' gut. Satisfactorily, FMT, a promising therapy for Clostridium difficile-associated diarrhea, has the high cure rate of diarrhea (Kelly et al., 2014b; Gough et al., 2011). However, some problems should be further solved and perfected, such as whether FMT should be used as a first-line therapy for the patients with Clostridium difficile-associated diarrhea. (iii) Case series: There are many different treatments for CDI and IBD, but none of the treatments are proved to be very useful. In order to find the solution that worked best, it was necessary to analyze representative cases. Rubin et al (Rubin et al., 2013) found the effectiveness of FMT in the therapy of CDI and IBD from a case series of 75 FMT course. This gave the future researchers a clue that case series would be an appropriate way.

There are some limitations to the current study. Non-English publications were excluded and as landmark articles published in other languages were not considered. The study only focused on the publications in WoSCC database, and the exclusion of other databases, such as PubMed and Scopus, may have produced slightly different results. Nevertheless, WoSCC is a comprehensive and popular Web database in the field of scientometrics (Miao et al., 2017b).

\section{CONCLUSIONS}

In conclusion, based on the detailed analysis of the distribution and changes in countries, citations, references, journals, authorship, institutions and keywords, the results of this research and analysis 
378 indicate that FMT is an area of very active research. This analysis using bibliometric methods

379 provides a solid overview of current FMT research and may help in providing guidance for further

380 studies.

381

382 FIGURE LEGENDS

383 Figure 1 Overview of article selection process.

384 Figure 2 The number of annual publications on FMT from 2004 to 2017(A), growth trends

of countries on FMT from 2004 to 2017(B), the cooperation of countries/regions contributed

to publications on FMT from 2004 to 2017(C), growth trend of keywords on FMT from 2004

to $2017(D)$.

Figure 3 Correlations between the number of citations and number of countries (A); the

number of institutions (B); the number of the years since publication(C); the number of

authors (D).

391

Figure 4 Reference co-citation map of publications (A); Network map of authors

392

contributed to publications (B); Network map of co-cited authors contributed to

393

publications (C); Network map of institutions contributed to publications (D) on FMT

from 2004 to 2017.

395

Figure 5 Visualization of two-dimensional knowledge map of keywords for the complete

dataset. 
399 FMT fecal microbiota transplantation

400 CDI Clostridium difficile infection

401 IBD inflammatory bowel disease

402 SCI-E the Science Citation Index Expanded

403 WoSCC the Web of Science Core Collection

404 T100 the top 100

405 JCR the Journal Citation Reports

406 ACKNOWLEDGEMENTS

407 The authors would like to thank editors and the anonymous reviewers for their valuable comments

408 and suggestions to improve the quality of the paper.

409

410

411 Supplemental Information

412 Figure S1. Correlations between citation index (WoSCC) and time cited (WOSCC) (A); citation 413 index (Scopus) and time cited (Scopus) (B); citation index (Scopus) and citation index (WoSCC)

414 (C).

415 Figure S2 Network visualization two-dimensional knowledge map of keywords for the complete

416 dataset.

417 Table S1. The top 100 most-cited articles ranked by the number of times cited

418 Table S2. Major clusters of co-cited references.

419 Table S3. Journals with the top 100 articles ranked by the number of citations. 


\section{REFERENCES}

422

Anderson JL, Edney RJ, and Whelan K. 2012. Systematic review: faecal microbiota transplantation in the management of inflammatory bowel disease. Aliment Pharmacol Ther 36:503-516. DOI:10.1111/j.1365-2036.2012.05220.x.

Brandt LJ, Aroniadis OC, Mellow M, Kanatzar A, Kelly C, Park T, Stollman N, Rohlke F, and Surawicz C. 2012. Long-Term Follow-Up of Colonoscopic Fecal Microbiota Transplant for Recurrent Clostridium difficile Infection. Am J Gastroenterol 107:1079-1087. DOI: 10.1038/ajg.2012.60.

Chen C, Ibekwe-Sanjuan F, and Hou J. 2010. The structure and dynamics of cocitation clusters: A multiple-perspective cocitation analysis. J Am Soc Inf Sci Technol 61:1386-1409. DOI: 10.1002/asi.21309.

Rousseau, R. 2014. Library science: Forgotten founder of bibliometrics. NATURE 510:218. DOI: $10.1038 / 510218 \mathrm{e}$.

Crowther M, Lim W, and Crowther MA. 2010. Systematic review and meta-analysis methodology. BLOOD 116:3140-3146. DOI:10.1182/blood-2010-05-280883.

Keathley-Herring H, Aken EV, Gonzalez-Aleu F, Deschamps F, Letens G, and Orlandini PC. 2016. Assessing the maturity of a research area: bibliometric review and proposed framework. SCIENTOMETRICS 109:927-951. DOI: 10.1007/s11192-016-2096-x.

Choi JE, and Kim MS. 2018. Exploring the Knowledge Structure of Nursing Care for Older Patients With Delirium: Keyword Network Analysis. Comput Inform Nurs 36: 216-224. DOI: 10.1097/CIN.0000000000000424.

Cohen NA, Ben AR, Guzner-Gur H, Santo ME, Halpern Z, and Maharshak N 2015. Fecal Microbiota Transplantation for Clostridium difficile-Associated Diarrhea. Israel Medical Association Journal Imaj 17:510-514.

Fu LD, and Aliferis C. 2008. Models for predicting and explaining citation count of biomedical articles. AMIA ... Annual Symposium proceedings. AMIA Symposium 6:222.

Will C. 2014. Citation counts and indices: Beware of bad data. PHYSICS TODAY 67:10-11. DOI: 10.1063/PT.3.2463.

Prathap, G. 2012. The inconsistency of the H - index. Journal of the American Society for Information Science \& Technology 63:1480-1481. DOI: 10.1002/asi.21678.

Garfield E. 2003. The Agony and the Ecstasy - The History and Meaning of the Journal Impact Factor. International Journal of Clinical \& Health Psychology. 295 :90-93.

Gough E, Shaikh H, and Manges AR. 2011. Systematic Review of Intestinal Microbiota Transplantation (Fecal Bacteriotherapy) for Recurrent Clostridium difficile Infection. Clin Infect Dis 53:994. DOI :10.1093/cid/cir632.

Halpenny D, Burke J, Mcneill G, Snow A, and Torreggiani WC. 2010. Geographic Origin of Publications in Radiological Journals as a Function of GDP and Percentage of GDP Spent on Research. Acad Radiol 17:768. DOI: 10.1016/j.acra.2010.01.020. 
459 Hamilton MJ, Weingarden AR, Sadowsky MJ, and Khoruts A. 2012. Standardized frozen 460 preparation for transplantation of fecal microbiota for recurrent Clostridium difficile infection. $\mathrm{Am}$ 461 J Gastroenterol 107:761. DOI: 10.1038/ajg.2011.482.

462 Hourigan SK, Chen LA, Grigoryan Z, Laroche G, Weidner M, Sears CL, and Oliva-Hemker 463 M. 2015. Microbiome changes associated with sustained eradication of Clostridium difficile after 464 single faecal microbiota transplantation in children with and without inflammatory bowel disease. 465 Gastroenterology 42:45. DOI: 10.1111/apt.13326.

466 Kappelman MD, Rifas-Shiman SL, Porter CQ, Ollendorf DA, Sandler RS, Galanko JA, and 467 Finkelstein JA. 2008. Direct Health Care Costs of Crohn's Disease and Ulcerative Colitis in US 468 Children and Adults. Gastroenterology 135:1907. DOI: 10.1053/j.gastro.2008.09.012.

469 Kelly CR, De LL, and Jasutkar N. 2012. Fecal microbiota transplantation for relapsing 470 Clostridium difficile infection in 26 patients: methodology and results. J Clin Gastroenterol 471 46:145. DOI: 10.1097/MCG.0b013e318234570b.

472 Kelly CR, Ihunnah C, Fischer M, Khoruts A, Surawicz C, Afzali A, Aroniadis O, Barto A, 473 Borody T, and Giovanelli A. 2014. Fecal microbiota transplant for treatment of Clostridium 474 difficile infection in immunocompromised patients. Am J Gastroenterol 109:1065-1071. DOI: 475 10.1038/ajg.2014.133.

476 Kelly CR, Khoruts A, Staley C, Sadowsky MJ, Abd M, Alani M, Bakow B, Curran P, 477 Mckenney J, and Tisch A. 2016. Effect of Fecal Microbiota Transplantation on Recurrence in 478 Multiply Recurrent Clostridium difficile InfectionA Randomized TrialFecal Microbiota 479 Transplantation and Recurrent $\mathrm{C}$ difficile Infection. Ann Intern Med. 22:18-19. DOI: 480 10.7326/M16-0271.

481 Khoruts A, Rank KM, Newman KM, Viskocil K, Vaughn BP, Hamilton MJ, and Sadowsky 482 MJ. 2016. Inflammatory Bowel Disease Affects the Outcome of Fecal Microbiota Transplantation 483 for Recurrent Clostridium difficile Infection. Clin Gastroenterol Hepatol 14:1433-1438. DOI: 484 10.1016/j.cgh.2016.02.018.

485 Khoruts A, Sadowsky MJ, and Hamilton MJ. 2015. Development of fecal microbiota 486 transplantation suitable for mainstream medicine. Clin Gastroenterol Hepatol 13:246-250. DOI: 487 10.1016/j.cgh.2014.11.014.

488 Konijeti GG, Sauk J, Shrime MG, Gupta M, and Ananthakrishnan AN. 2014. Cost489 effectiveness of Competing Strategies for Management of Recurrent Clostridium difficile Infection: A Decision Analysis. Clin Infect Dis 58:1507-1514. DOI: 10.1093/cid/ciu128.

Liu YH, Wang SQ, Xue JH, Liu Y, Chen JY, Li GF, He PC, and Tan N. 2016. Hundred topcited articles focusing on acute kidney injury: a bibliometric analysis. BMJ Open 6:e11630. DOI:10.1136/bmjopen-2016-011630.

Mcfarland LV. 2005. Alternative treatments for Clostridium difficile disease: what really works? J Med Microbiol 54:101-111. DOI: 10.1099/jmm.0.45753-0.

Merlo G, Graves N, and Connelly L. 2016. Economic Evaluation of Fecal Microbiota Transplantation for The Treatment of Recurrent Clostridium Difficile Infection In Australia. Value Health 31:1927. DOI: 10.1016/j.jval.2015.09.2215.

Miao Y, Liu R, Pu Y, and Yin L. 2017. Trends in esophageal and esophagogastric junction cancer 
500 research from 2007 to 2016: A bibliometric analysis. Medicine 96:e6924. 501 DOI:10.1097/MD.0000000000006924.

502 Nagalingam NA, and Lynch SV 2012. Role of the microbiota in inflammatory bowel diseases. 503 Inflamm Bowel Dis 18:968. DOI: 10.1002/ibd.21866.

504 Orenstein R, Griesbach CL, and Dibaise JK. 2013. Moving fecal microbiota transplantation into the mainstream. Nutr Clin Pract 28:589-598. DOI: 10.1177/0884533613497516.

506 Zhao SX, Yu S, Tan AM, Xu X, and Yu H. 2016. Global pattern of science funding in economics. 507

Ashok A, Damayanthi D, Sindhuja T, Sandro C Esteves, Avi Harlev, Ralf Henkel,

Shubhadeep Roychoudhury, Sheryl Homa, Nicolás Garrido Puchalt, Ranjith Ramasamy,

Ahmad Majzoub, Kim Dao Ly, Eva Tvrda, Mourad Assidi, Kavindra Kesari, Reecha

Sharma, Saleem Banihani, Edmund Ko, Muhammad Abu-Elmagd, Jaime Gosalvez, Asher

Bashiri. 2016. Bibliometrics: tracking research impact by selecting the appropriate metrics. Asian

J Androl, 8:296-309. DOI: 10.4103/1008-682X.171582.

CS. 2018. Bibliometric analysis of research on the role of intestinal microbiota in obesity. Peer J,

6: DOI: $10.7717 /$ peerj.5091.

Rodemann JF, Dubberke ER, Reske KA, Seo DH, and Stone CD. 2007. Incidence of Clostridium difficile infection in inflammatory bowel disease. Clin Gastroenterol Hepatol 5:339344. DOI: 10.1016/j.cgh.2006.12.027.

Rohlke F, and Stollman N. 2012. Fecal microbiota transplantation in relapsing Clostridium difficile infection. Therap Adv Gastroenterol 5:403-420. DOI: 10.1177/1756283X12453637. recurrent Clostridium difficile infection: report on a case series. Anaerobe 19:22-26. DOI: 10.1016/j.anaerobe.2012.11.004.

Suk FM, Lien GS, Yu TC, and Ho YS. 2011. Global trends in Helicobacter pylori research from 1991 to 2008 analyzed with the Science Citation Index Expanded. Eur J Gastroenterol Hepatol 23:295-301. DOI: 10.1097/MEG.0b013e3283457af7.

Su HN, and Lee PC. 2010. Mapping knowledge structure by keyword co-occurrence: a first look at journal papers in Technology Foresight. SCIENTOMETRICS 85:65-79. DOI: 10.1007/s11192530 010-0259-8.

531 Surawicz CM, Brandt LJ, Binion DG, Ananthakrishnan AN, Curry SR, Gilligan PH, 
532 Mcfarland LV, Mellow M, and Zuckerbraun BS. 2013. Guidelines for diagnosis, treatment, 533 and prevention of Clostridium difficile infections. Am J Gastroenterol 108:478-498. DOI: 534 10.1038/ajg.2013.4

535 Talley NJ, Abreu MT, Achkar JP, Bernstein CN, Dubinsky MC, Hanauer SB, Kane SV, 536 Sandborn WJ, Ullman TA, and Moayyedi P. 2011. An evidence-based systematic review on 537 medical therapies for inflammatory bowel disease. Am J Gastroenterol 106 Suppl 1:S2-25. DOI: 538 10.1038/ajg.2011.58.

539 van Nood E, A V, M N, S F, EG Z, de Vos WM, CE V, EJ K, JF B, and JG T. 2013. Duodenal 540 infusion of donor feces for recurrent Clostridium difficile. N. Engl. J. Med. 368:407-415. DOI:

541 10.1056/NEJMoa1205037.

542 Van Rossum M, Bosker BH, Pierik EGJM, and Verheyen CCPM. 2010. Geographic origin of 543 publications in surgical journals. Br J Surg 94:244-247. DOI: 10.1002/bjs.5571.

544 Yan R, Tang J, Liu X, Shan D, and Li X. 2011. Citation count prediction: learning to estimate 545 future citations for literature. The 20th ACM CIKM 2011 conference 2011:1247-1252. DOI: $54610.1145 / 2063576.2063757$.

547 Yin Z, Chen D, and Li B. 2016. Global Regulatory T-Cell Research from 2000 to 2015: A 548 Bibliometric Analysis. PLoS One 11:e162099. DOI: 10.1371/journal.pone.0162099.

549 Zhang F, Luo W, Shi Y, Fan Z, and Ji G. 2012. Should we standardize the 1,700-year-old fecal 550 microbiota transplantation? Am J Gastroenterol 107:1755-1756. DOI: 10.1038/ajg.2012.251. 
Figure 1

Overview of article selection process.

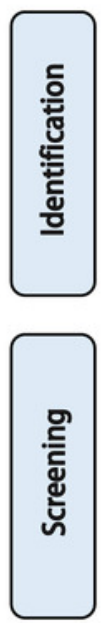

Keywords and free words search in Web of Science Core Collection (WoSCC) $(n=2062)$

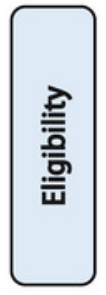

Titles and abstract screened $(n=824)$

Titles and abstracts of articles excluded $(n=1238)$

Titles and abstracts unrelated to the study $(n=809)$

The main content unrelated to the study $(n=125)$

The abstract inaccessible $(n=302)$

Duplicates $(n=2)$

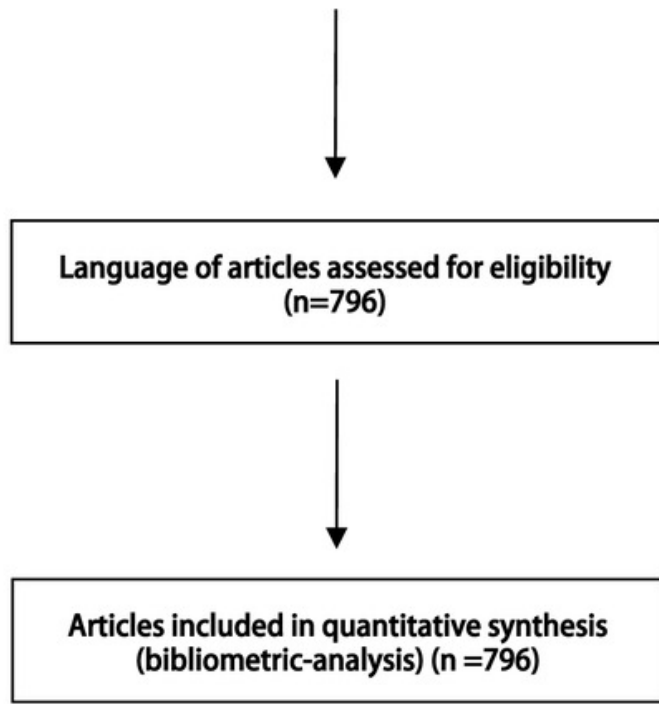

Language containing non-English $(n=28)$ 
Figure 2

The number of annual publications $(A)$, and growth trends of countries(B), the cooperation of countries/regions(C), growth trend of keywords(D) on FMT from 2004 to 2017.

A
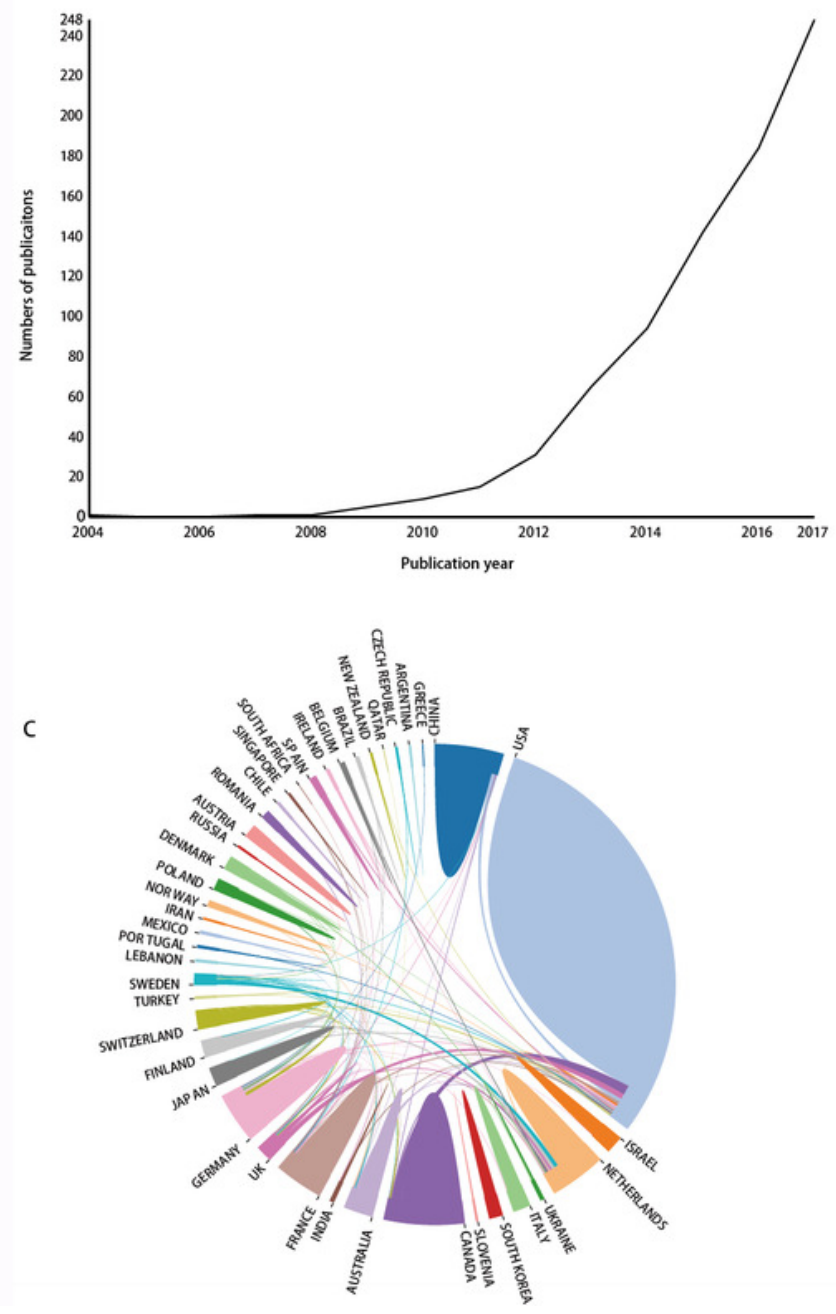

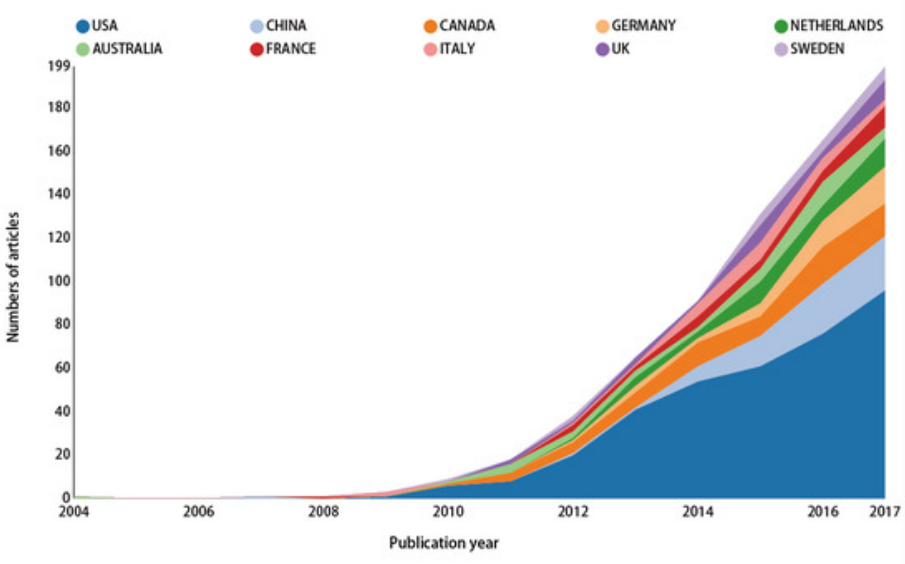

D

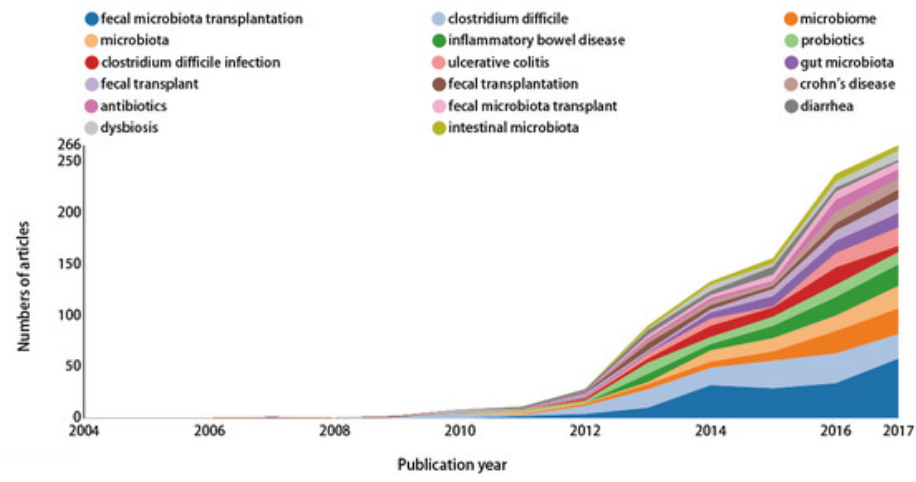


Figure 3

Correlations between the number of citations and countries $(A)$, institutions $(B)$, the years since publication(C), and authors (D).
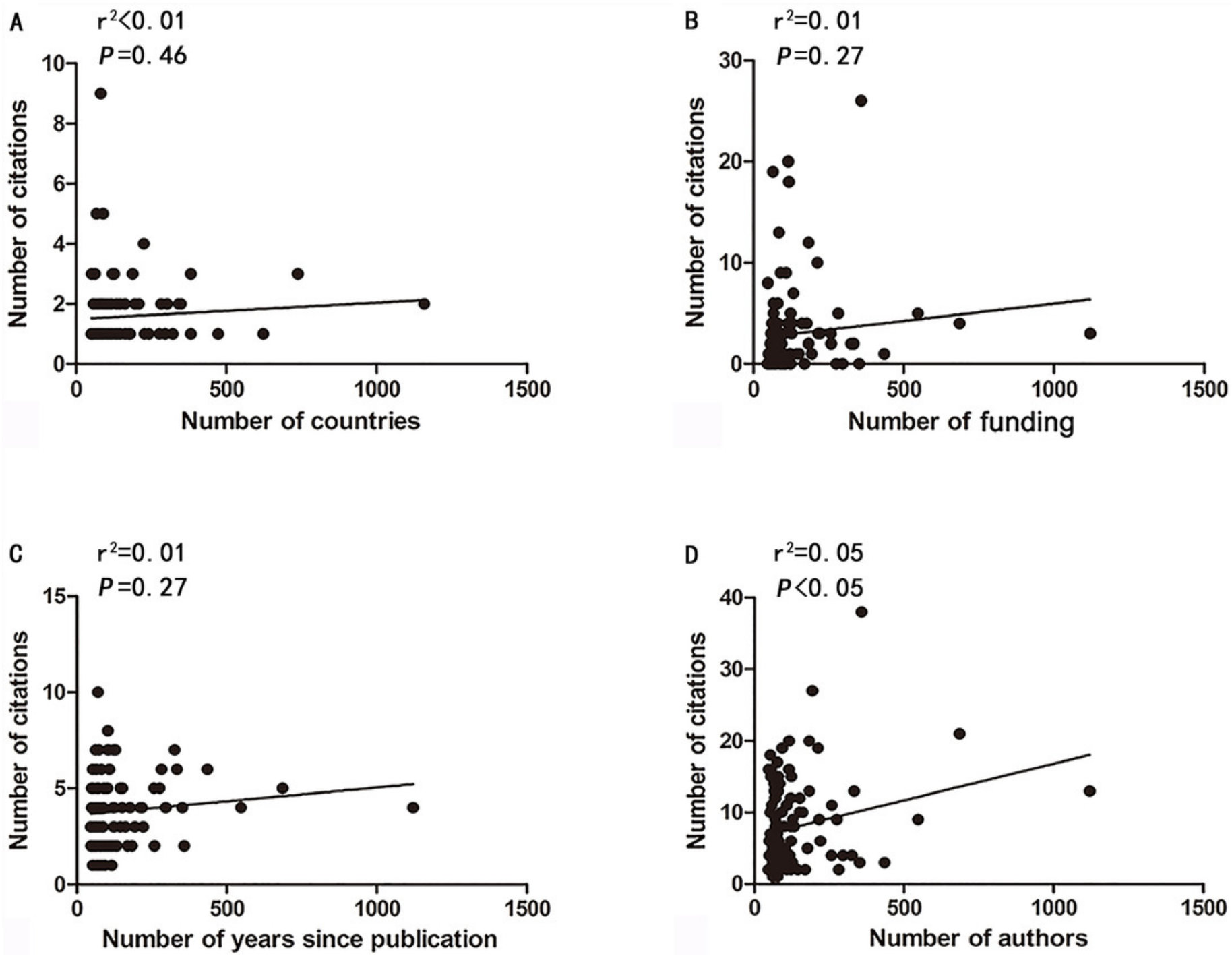


\section{Figure 4}

\section{Reference co-citation map(A), Network map of authors (B), Network map of co-cited authors (C) and Network map of institutions (D) contributed to publications on FMT from 2004 to 2017.}

A

modularity $Q=0.54$
silhouetere sore $=0.32$

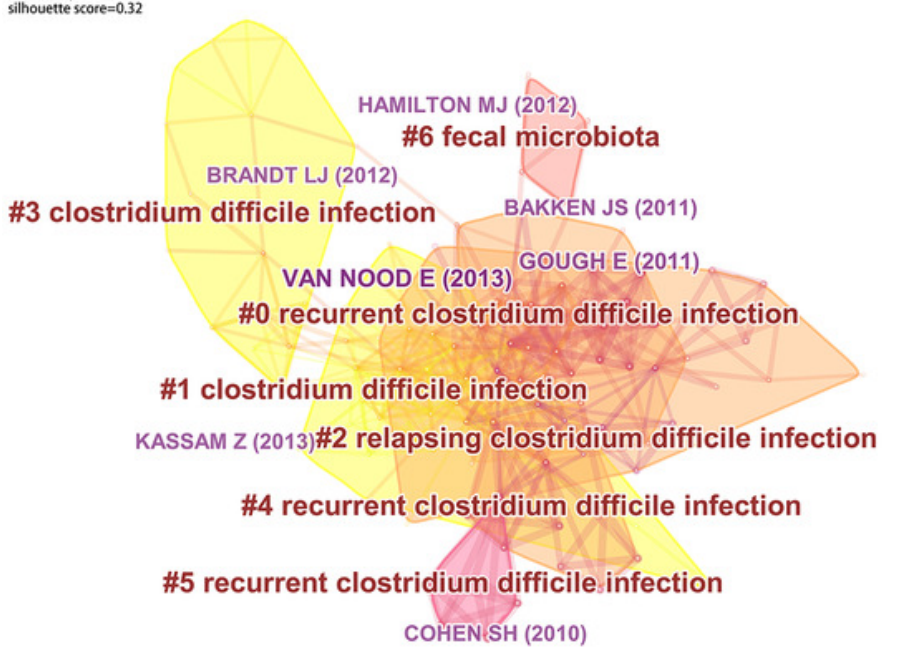

C

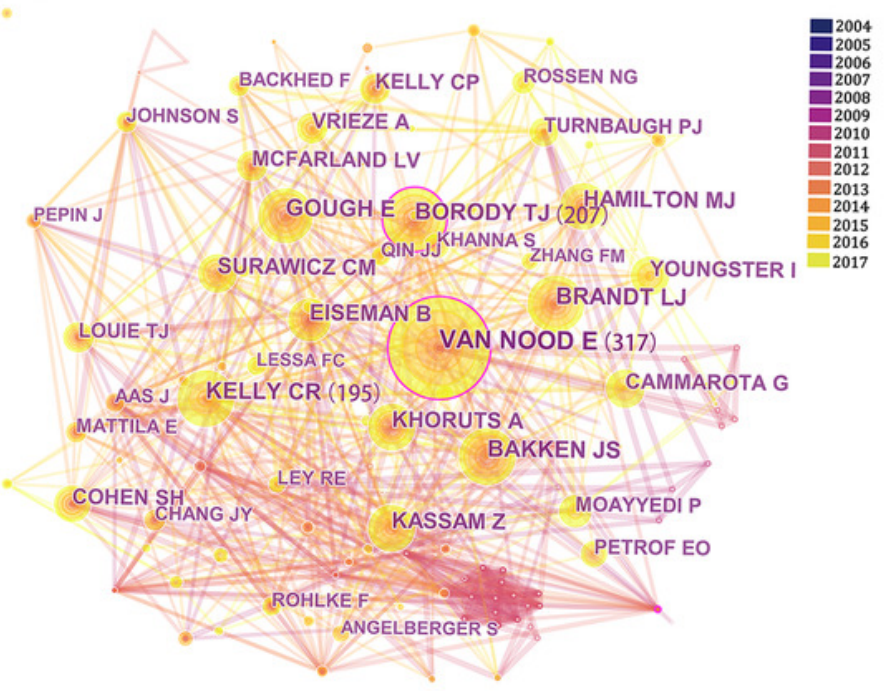

B

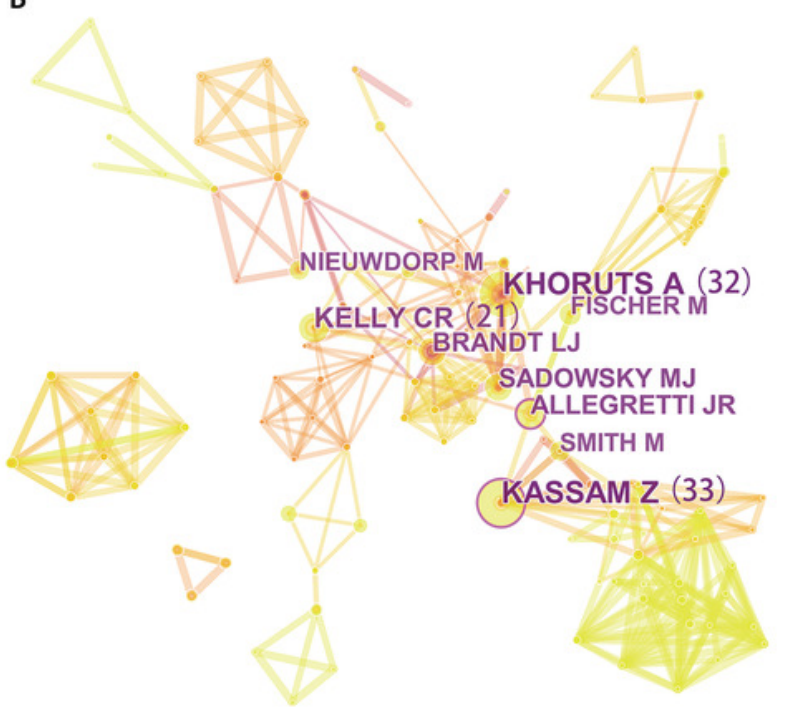

D

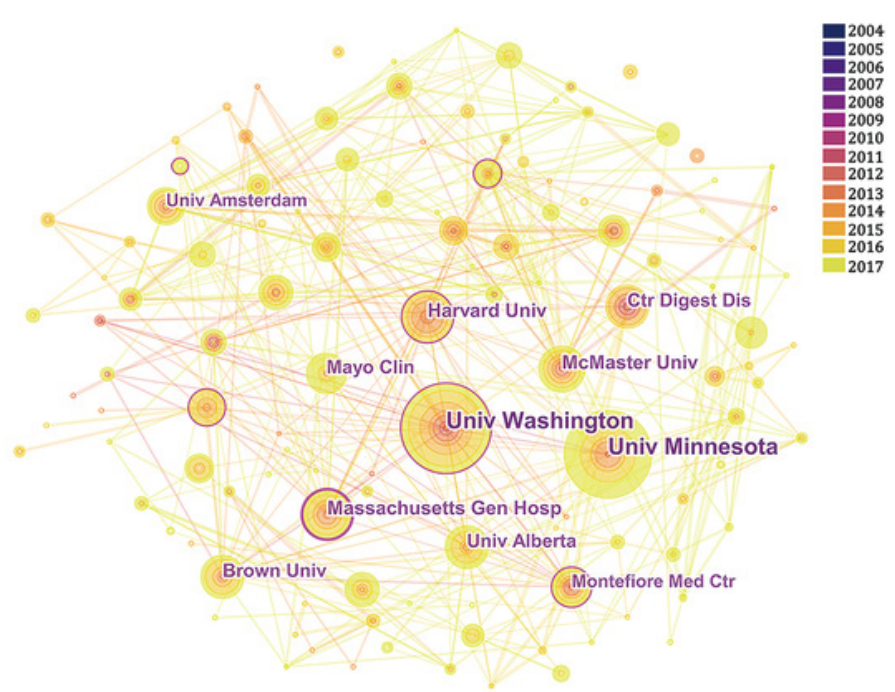




\section{Figure 5}

\section{Visualization of two-dimensional knowledge map of keywords for the complete dataset.}

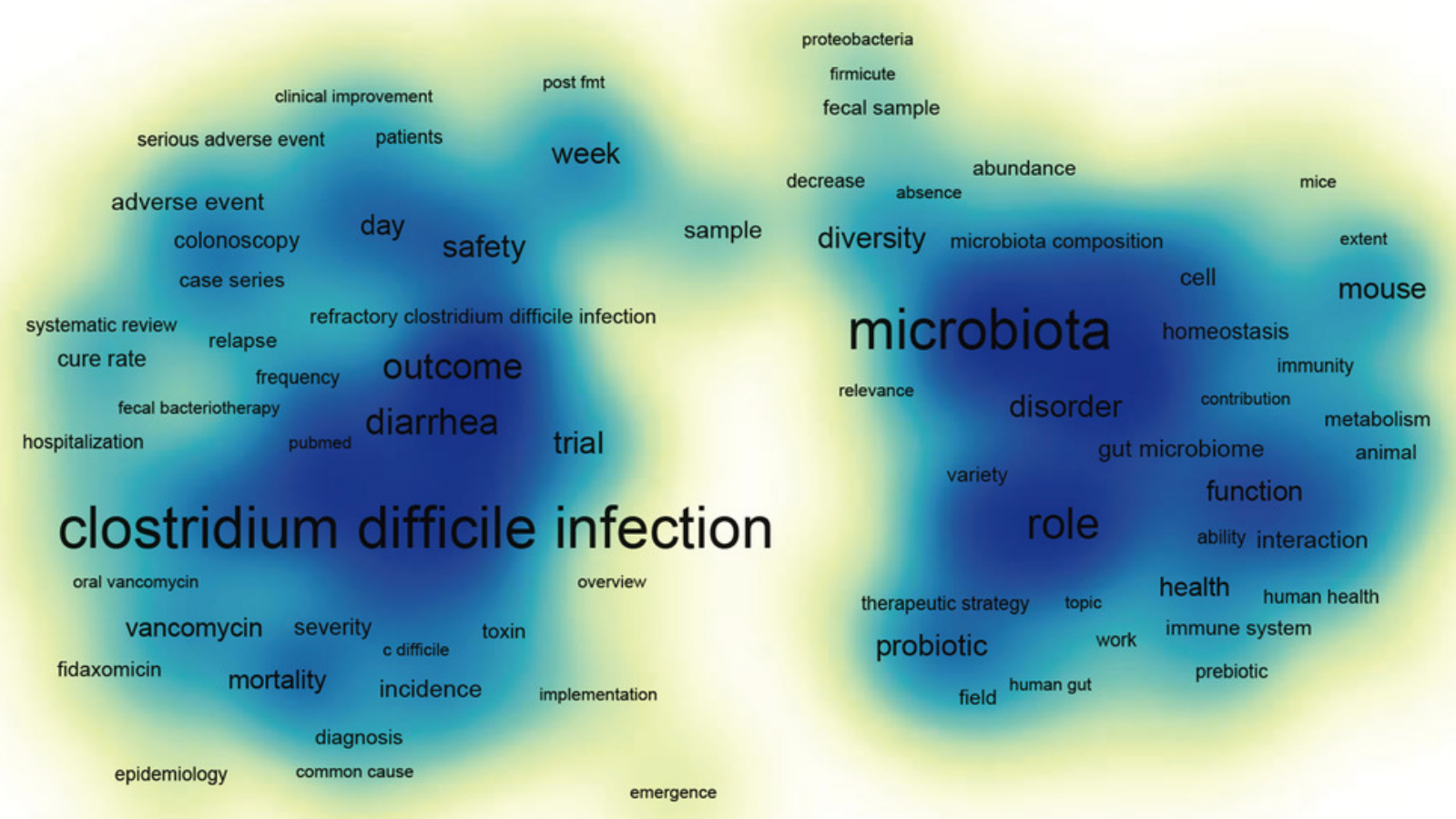




\section{Table $\mathbf{1}$ (on next page)}

Bibliometric information associated with the top 5 of the top 100 cited articles in FMT from 2004 to 2017 
Table 1. Bibliometric information associated with the top 5 of the top 100 cited articles in FMT from 2004 to 2017

Rank Title

Years Times cited Citation index Time cited Citation index

\begin{tabular}{llllll} 
& & (WoSCC) & (WoSCC) & (Scopus) & (Scopus) \\
\hline 1 Van Nood E, Vrieze A, Nieuwdorp M, et al. Duodenal 2013 & 1158 & 231.60 & 1307
\end{tabular}

infusion of donor feces for recurrent Clostridium difficile

[J]. N Engl J Med,2013,368(5):407-415.

2 Vrieze A, Van N E, Holleman F, et al. Transfer of intestinal

microbiota from lean donors increases insulin sensitivity in individuals with metabolic syndrome [J]. Gastroenterology, 2012, 143(4):913-916.

diagnosis, treatment, and prevention of Clostridium difficile infections [J]. American Journal of Gastroenterology, 2013, 108(4):478-498. 
intestinal microbiota transplantation (fecal bacteriotherapy) for recurrent Clostridium difficile infection $[\mathrm{J}]$. Clinical Infectious Diseases An Official Publication of the Infectious Diseases Society of America, 2011, 53(10):994.

5 Vétizou M, Pitt J M, Daillère R, et al. Anticancer 2015

immunotherapy by CTLA-4 blockade relies on the gut microbiota [J]. Science, 2015, 350(6264):1079. 


\section{Table 2 (on next page)}

The top 5 journals of the top 100 articles ranked by the number of citation contributed to publications on FMT from 2004 to 2017. 
Table 2. The top 5 journals of the top $\mathbf{1 0 0}$ articles ranked by the number of citation contributed to publications on FMT from 2004 to 2017.

\begin{tabular}{lllll} 
Rank & Journal & Country & No. of articles & IF 2017 \\
\hline 1 & AMERICAN JOURNAL OF & the US & 10 & 10.231 \\
& GASTROENTEROLOGY & & \\
2 & GASTROENTEROLOGY & the US & 8 & 20.773 \\
3 & JOURNAL OF CLINICAL & the US & 8 & 2.975 \\
4 & GASTROENTEROLOGY & & & \\
5 & CLINICAL INFECTIOUS DISEASES & the US & 4 & 9.117 \\
5 & CELL & the US & 3 & 31.398
\end{tabular}

1 


\section{Table 3 (on next page)}

The top 5 authors ranked by the number of articles 
Table 3. The top 5 authors ranked by the number of articles

\begin{tabular}{llllllll} 
Rank & Authors & No. of & Total & First & Citations & Correspond & Citations of \\
& & articles & citations & of first & correspond \\
\hline 1 & Kassam, Z & 38 & 325 & 4 & 186 & 1 & 7 \\
2 & Khoruts, A & 33 & 755 & 6 & 153 & 9 & 202 \\
3 & Kelly, CR & 24 & 375 & 5 & 243 & 8 & 297 \\
4 & Sadowsky, MJ & 23 & 405 & 2 & 0 & 6 & 148 \\
5 & Allegretti, JR & 22 & 22 & 5 & 4 & 1 & 0 \\
\hline
\end{tabular}




\section{Table 4 (on next page)}

The top 5 authors ranked by the number of citations 
Table 4. The top 5 authors ranked by the number of citations

\begin{tabular}{llllllll} 
Rank & Authors & No. of & Total & First & Citations & Correspon & Citations of \\
& & articles & citations & of first & d & correspond \\
\hline 1 & Khoruts, A & 33 & 755 & 6 & 153 & 9 & 202 \\
2 & Brandt, LJ & 20 & 720 & 8 & 271 & 9 & 342 \\
3 & de Vos, WM & 8 & 557 & 0 & 0 & 0 & 0 \\
4 & Nieuwdorp, M & 18 & 509 & 0 & 0 & 9 & 171 \\
5 & Zoetendal, EG & 4 & 472 & 0 & 0 & 0 & 0 \\
\hline
\end{tabular}




\section{Table 5 (on next page)}

The top 5 institutions ranked by the number of articles contributed to publications on FMT from 2004 to 2017. 
Table 5. The top 5 institutions ranked by the number of articles contributed to publications on FMT from 2004 to 2017.

Rank Institution $\quad$ No. of articles No. of citations

\begin{tabular}{llll}
\hline 1 & University of Minnesota, Gemini, Minnesota, the US & 99 & 1999 \\
2 & University of washington, Seattle, Washington, the & 58 & 1136 \\
& US & & \\
3 & McMaster University, Hamilton, Ontario, Canada & 34 & 1099 \\
4 & Emory University, Atlanta, GA, the US & 32 & 58 \\
5 & University of Alabama Birmingham, Birmingham, & 30 &
\end{tabular}


Table 6(on next page)

Core Keywords related to FMT from 2004 to 2017. 
Table 6. Core Keywords related to FMT from 2004 to 2017.

\begin{tabular}{|c|c|c|c|}
\hline Domain & Topic & Percentage Within & Frequency of Keyword Occurrence (n) \\
\hline & & Core Keywords, \% & \\
\hline
\end{tabular}

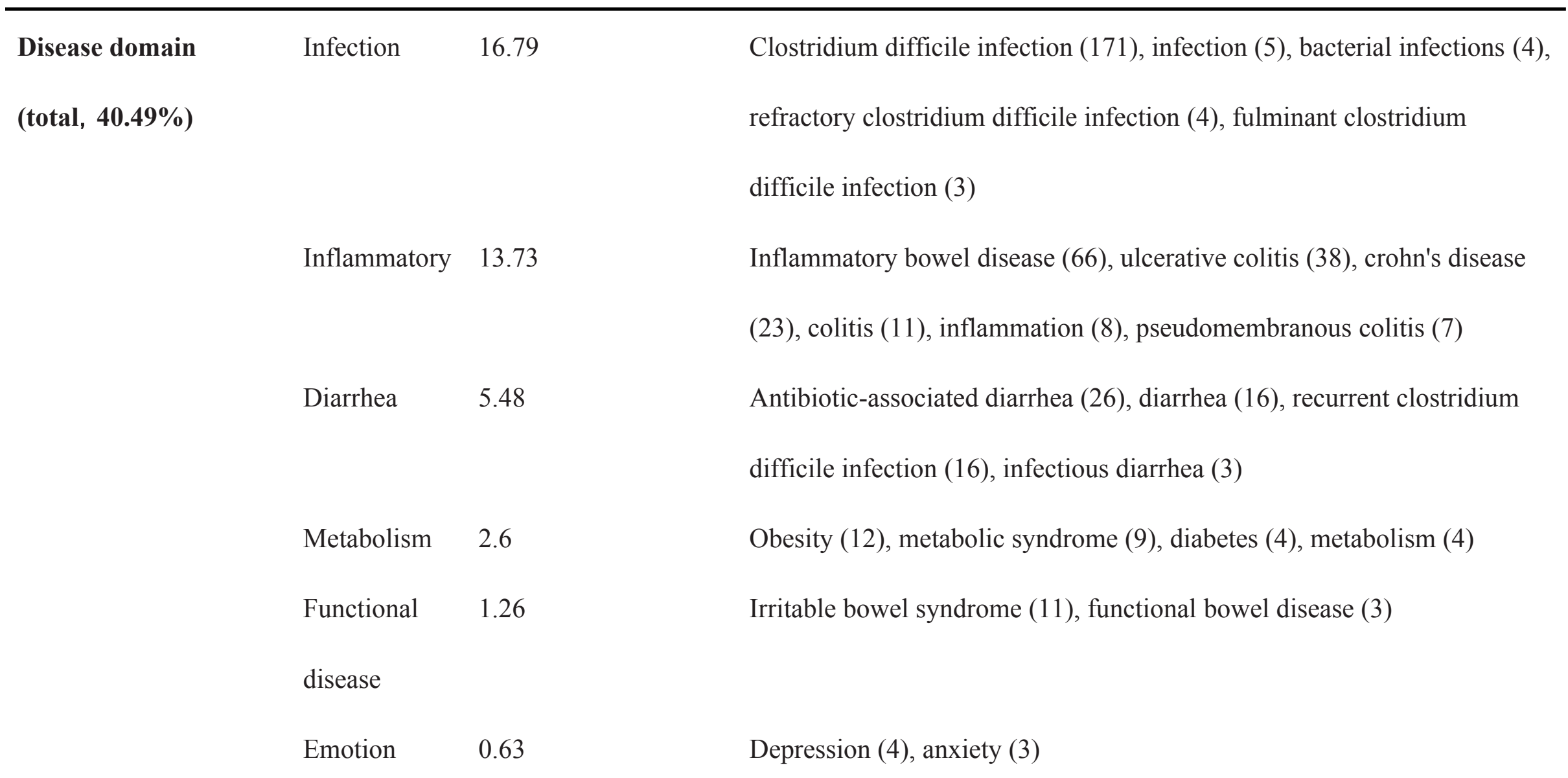




\section{Nosogenesis domain}

Microbiota

11.13

(total, $14.63 \%)$

Environment

Trial domain

(total, 3.77\%)

Method

Therapy domain

Transplantati

(total, 41.44\%) on

Food

Antibiotics
Gut microbiota (49), microbiota (40), intestinal microbiata (27),

bifidobacterium (5), lactobacillus (3)

Dysbiosis (17), gut (4), rain-gut axis (3), gastrointestinal (3), gut

permeability (3)

Immunomodulation (9)

Children (13), germ-free (5), germ-free animals (3), mathematical

modeling (3), mouse models (3)

16s rRNA analysis (8), metagenomics (4), clinical trial (3)

Fecal microbiota transplantation (301), microbiome (41), stool

transplantation (9), bacterial consortium transplantation (7),

bacteriotherapy (6), gut microbiome transplantation (4), transplantation

(3)

Probiotics (33), prebiotic (8), diet (5)

Antibiotic therapy (13), fidaxomicin (6), vancomycin (6), metronidazole 


\section{PeerJ}

(4), infection control (3)

$\begin{array}{lll}\text { Fibroptic } & 0.81 \quad \text { Colonoscopy (9) }\end{array}$

endoscopy 
Table 7 (on next page)

The top 7 Keywords with the Strongest Citation Bursts on FMT from 2004 to 2017 
Table 7. The top 7 Keywords with the Strongest Citation Bursts on FMT from 2004 to 2017

\begin{tabular}{|c|c|c|c|c|c|}
\hline Keywords & Year & Strength & Begin & End & $2004-2017$ \\
\hline flora & 2004 & 10.5588 & 2011 & 2014 & \\
\hline & & & & & 든 \\
\hline bacteriotherapy & 2004 & 7.6856 & 2009 & 2013 & سم \\
\hline diarrhea & 2004 & 7.0221 & 2011 & 2013 & سم \\
\hline case series & 2004 & 6.2972 & 2011 & 2013 & سمس - سـ \\
\hline antibiotic associated & 2004 & 5.4075 & 2010 & 2013 & سمس \\
\hline diarrhea & & & & & \\
\hline coliti & 2004 & 5.0701 & 2010 & 2013 & س \\
\hline
\end{tabular}


